\title{
POTENCIAL FORRAJERO DE CULTIVARES DE CANOLA PRIMAVERALES E INVERNALES EN LA COMARCA LAGUNERA, MÉXICO
}

\author{
FORAGE POTENTIAL OF SPRING AND WINTER CANOLA CULTIVARS \\ IN THE COMARCA LAGUNERA REGION, MEXICO
}

\section{David G. Reta-Sánchez ${ }^{1}$ *, J. Santos Serrato-Corona², Héctor M. Quiroga-Garza', Uriel Figueroa-Viramontes ${ }^{1}$ y Arturo Gaytán-Mascorro'}

\begin{abstract}
'Campo Experimental La Laguna, Instituto Nacional de Investigaciones Forestales, Agrícolas y Pecuarias. Blvd. José Santos Valdez 1200.27440, Col. Centro, Cd. Matamoros, Coahuila. Tel. 01-800-088-2222 Ext. 82415. Facultad de Agricultura y Zootecnia, Universidad Juárez del Estado de Durango. Domicilio Conocido, Ejido Venecia, Dgo. Apdo. Postal 1-142. Gómez Palacio, Dgo.
\end{abstract}

*Autor para correspondencia (reta.david@inifap.gob.mx)

\section{RESUMEN}

\begin{abstract}
La adecuada selección del tipo y cultivar de canola (Brassica napus L.) para forraje puede incrementar los rendimientos de materia seca (MS) y nutrientes. El objetivo del estudio fue evaluar el potencial forrajero de cultivares de canola tipo primaveral e invernal en la Comarca Lagunera, México. Dos experimentos se realizaron en Matamoros, Coahuila, México, durante los ciclos agrícolas 2012-2013 y 2013-2014. Se evaluaron seis cultivares primaverales y tres invernales, la variedad IMC 205 y el híbrido Hyola 401 fueron utilizados como testigos. El diseño experimental fue de bloques completos al azar con cuatro repeticiones. Los cultivares sobresalientes con mayor rendimiento de MS y nutrientes $(P \leq 0.05)$ fueron los testigos, y los nuevos cultivares Monty, Claremore y especialmente Ortegón, que presentó la ventaja adicional de una mayor precocidad a la cosecha (de 6 a 16 d) respecto a los testigos. El rendimiento en los cultivares primaverales sobresalientes fue de 7216 a 8480 $\mathrm{kg} \mathrm{ha}^{-1}$ de MS, de 1270 a $1456 \mathrm{~kg} \mathrm{ha}^{-1}$ de proteína cruda (PC) y de 39,663 a $47,928 \mathrm{MJ} \mathrm{ha}^{-1}$ en energía neta para lactancia $\left(\mathrm{EN}_{\mathrm{L}}\right)$. El mejor cultivar invernal fue Claremore, con un rendimiento de MS inferior $(P \leq 0.05)$ a los cultivares primaverales ( $\left.6215 \mathrm{~kg} \mathrm{ha}^{-1}\right)$; sin embargo, debido a su mayor contenido de nutrientes, sus rendimientos de PC (1431 $\left.\mathrm{kg} \mathrm{ha}^{-1}\right)$ y EN (42,625 MJ ha-1) fueron similares a los obtenidos por los mejores cultivares primaverales. En conclusión, es posible incluir al menos tres nuevos cultivares de canola para la producción de forraje en la región.
\end{abstract}

Palabras clave: Brassica napus var. oleifera, composición química, rendimiento de materia seca, nutrientes.

\section{SUMMARY}

The adequate selection of type and cultivar of forage canola (Brassica napus L.) may increase dry matter (DM) and nutrient yields. This study evaluated the forage potential of Spring and Winter canola cultivars in the Comarca Lagunera, Mexico. Two trials were carried out during the 20122013 and 2013-2014 agricultural cycles in Matamoros, Coahuila, Mexico. Six Spring and three Winter cultivars were evaluated; the IMC 205 variety and the hybrid Hyola 401 were used as controls. A randomized complete blocks design with four replications was used. The outstanding cultivars with higher DM and nutrient yields $(P \leq 0.05)$ were the controls and the new cultivars Monty, Claremore, and Ortegon. The Ortegon cultivar had particularly higher yields and the additional advantage of earliness at harvest (from 6 to $16 \mathrm{~d}$ ) compared to the control cultivars. Yield of the outstanding spring cultivars was 7216 to $8480 \mathrm{~kg} \mathrm{ha}^{-1} \mathrm{DM}, 1270$ to $1456 \mathrm{~kg} \mathrm{ha}^{-1}$ of crude protein (CP) and 39,663 to $47,928 \mathrm{MJ} \mathrm{ha}^{-1}$ in net energy for lactation $\left(\mathrm{NE}_{\mathrm{L}}\right)$. The best Winter cultivar was Claremore, with a DM yield $\left(6215 \mathrm{~kg} \mathrm{ha}^{-1}\right)$ lower $(P \leq 0.05)$ than the outstanding Spring cultivars; however, its yields in CP $\left(1431 \mathrm{~kg} \mathrm{ha}^{-1}\right)$ and $\mathrm{NE}_{\mathrm{b}}\left(42,625 \mathrm{MJ} \mathrm{ha}^{-1}\right)$ were similar to values from the best spring cultivars, due to its higher nutrient content. In conclusion, it is possible to include at least three new canola cultivars for forage production in the region.

Index words: Brassica napus var. oleifera, chemical composition, dry matter yield, nutrients.

\section{INTRODUCCIÓN}

La producción de leche de ganado bovino es una de las principales actividades económicas en la Comarca Lagunera, México. La alimentación del ganado se basa en forrajes irrigados como alfalfa (Medicago sativa L.), maíz (Zea mays L.), sorgo (Sorghum bicolor L. Moench) y avena (Avena sativa L.); sin embargo, la baja disponibilidad de agua, los crecientes problemas de salinidad en el suelo y en el agua, y las altas temperaturas ambientales restringen el potencial forrajero de los cultivos (Reta et al., 2008). Por ello, es importante identificar cultivos alternativos que contribuyan a incrementar la eficiencia en el uso del agua y producción de forraje.

Tradicionalmente, la canola (Brassica napus L.) se cultiva para producir aceite comestible; sin embargo, también produce forraje de buena calidad con rendimientos de materia seca (MS) entre 8906 y 10,300 kg ha-1 bajo irrigación y una productividad del agua (PA) superior a la reportada en avena, uno de los principales forrajes tradicionales (Cruz et al., 2012; Reta et al., 2008; Reta et al., 2015). Además, la canola es tolerante a bajas temperaturas (Fiebelkorn y Rahman, 2016), salinidad de los suelos (Purty et al., 2008) y es precoz para la producción de forraje (Reta et al., 2008) 
lo que incrementa su potencial como cultivo alternativo. No obstante, como nueva alternativa forrajera, el cultivo de canola aún requiere de desarrollo tecnológico adecuado para su producción; es importante, entre otros aspectos, seleccionar el tipo de cultivar a utilizar considerando las condiciones ambientales del área. En México se ha generado información sobre el potencial de rendimiento de grano de diversos cultivares de canola extranjeros, y de cultivares liberados en México; a pesar de ello, la información sobre su potencial forrajero aún es limitada.

En México se ha determinado el potencial de rendimiento de semilla de híbridos y variedades de canola tipo primaveral introducidas de Canadá, Europa, Australia y Estados Unidos; además de cultivares generados en México por el Instituto Nacional de Investigaciones Forestales, Agrícolas y Pecuarias (INIFAP) a partir del ciclo otoño-invierno 20052006. De los cultivares extranjeros evaluados sobresalen el híbrido Hyola 401, con rendimientos de 1698 a 3477 kg $\mathrm{ha}^{-1}$, y las variedades Monty e IMC 205 con rendimientos de 1417 a $3018 \mathrm{~kg} \mathrm{ha}^{-1}$ y de 1380 a $2456 \mathrm{~kg} \mathrm{ha}^{-1}$, respectivamente (García et al., 2013; Hernández et al., 2008; Hernández et al., 2009; Ortegón et al., 2006; Ortegón-Morales et al., 2007). Entre los cultivares liberados en México se encuentran Aztecan, Canomex, Centenario, Mexicano, Canorte y Ortegón, los cuales muestran rendimientos de semilla de 2204 a $3053 \mathrm{~kg} \mathrm{ha}^{-1}$, y además son de 8 a $10 \mathrm{~d}$ más precoces que el híbrido Hyola 401 (García et al., 2013; González y Ortegón, 2013).

La producción de forraje de canola en México se ha estudiado principalmente en la Comarca Lagunera, a través de los cultivares primaverales Hyola 401 e IMC 205. De acuerdo con el manejo agronómico y condiciones ambientales, los rendimientos de MS fluctúan de 5311 a 10,300 $\mathrm{kg} \mathrm{ha}^{-1}$, con concentraciones de proteína cruda (PC) de 159.0 a $280.0 \mathrm{~g} \mathrm{~kg}^{-1}, 340$ a $433 \mathrm{~g} \mathrm{~kg}^{-1}$ de fibra detergente ácido (FDA), 389 a $466.0 \mathrm{~g} \mathrm{~kg}^{-1}$ de fibra detergente neutro (FDN) y 3.7 a $6.1 \mathrm{MJ} \mathrm{kg}^{-1} \mathrm{MS}$ en energía neta para lactancia $\left(E N_{L}\right)$. Los rendimientos de PC varían de 978 a $2486 \mathrm{~kg} \mathrm{ha}^{-1}$, mientras que los de $\mathrm{EN}_{\mathrm{L}}$ oscilan de 20,200 a 61,182 $\mathrm{MJ} \mathrm{ha}^{-1}$ (Cruz et al., 2012; Reta et al., 2008; Reta et al., 2010; Reta et al., 2015; Reta et al., 2016; Reta-Sánchez et al., 2016a, 2016b).

El comportamiento de cultivares invernales de canola como cultivo forrajero no se ha evaluado en México. En Australia se ha estudiado la respuesta de estos cultivares con doble propósito, para obtener una cosecha de forraje antes del invierno en el año de siembra, y una cosecha de grano el siguiente año durante primavera-verano. Este sistema permite la producción de forraje entre 2500 y 6800 kg MS ha-1, y de 2500 a $5000 \mathrm{~kg} \mathrm{ha}^{-1}$ de grano (Paridaen y Kikegaard, 2015; Sprague et al., 2015). Con este mismo sistema de producción, Neely et al. (2009) encontraron en Estados Unidos de América (E.E.U.U.) que los cultivares invernales produjeron tres cosechas de forraje, con un promedio de $2100 \mathrm{~kg} \mathrm{MS} \mathrm{ha}^{-1}$ en cada corte. El objetivo de este estudio fue evaluar el potencial forrajero de cultivares de canola de tipo primaveral e invernal bajo las condiciones ambientales de la Comarca Lagunera de México.

\section{MATERIALES Y MÉTODOS}

El estudio se realizó en el Campo Experimental La Laguna (CELALA) del Instituto Nacional de Investigaciones, Forestales, Agrícolas y Pecuarias (INIFAP), localizado en Matamoros, Coahuila, México ( $25^{\circ} 32^{\prime}$ latitud N, $103^{\circ} 14^{\prime}$ longitud 0 y $1150 \mathrm{msnm}$ ), en un suelo arcillo-limoso. La preparación de la cama de siembra se realizó con un paso de arado a $0.3 \mathrm{~m}$ de profundidad, seguido de doble rastreo y nivelación.

La dosis de fertilización de N y P se calculó considerando su disponibilidad en el suelo y la capacidad de extracción de canola para un rendimiento de MS de $8132 \mathrm{~kg}$ ha${ }^{1}$, con una concentración en el forraje de $32.0 \mathrm{~g} \mathrm{~kg}^{-1}$ de N (Reta et al., 2008; Reta et al., 2010; Reta et al., 2015; Reta et al., 2016a) y $3.0 \mathrm{~g} \mathrm{~kg}^{-1}$ de P (Brennan y Bolland, 2001). Los requerimientos estimados del cultivo fueron de $260 \mathrm{~kg} \mathrm{~N}$ $\mathrm{ha}^{-1}$ y $55 \mathrm{~kg} \mathrm{P}_{2} \mathrm{O}_{5} \mathrm{ha}^{-1}$. Al considerar que el análisis de suelo a una profundidad de $0.3 \mathrm{~m}$ indicó una disponibilidad por hectárea de $40.4 \mathrm{~kg}$ de $\mathrm{N}$ y $28.4 \mathrm{~kg}$ de $\mathrm{P}_{2} \mathrm{O}_{5}$, se aplicó una dosis total de $240 \mathrm{~kg} \mathrm{~N} \mathrm{ha}^{-1}$ y $60 \mathrm{~kg}$ de $\mathrm{P}_{2} \mathrm{O}_{5}$ para cubrir los requerimientos del cultivo. Antes de la siembra, cada parcela experimental se fertilizó en forma manual con 75 $\mathrm{kg} \mathrm{N} \mathrm{ha}^{-1}$ (sulfato de amonio) y $60 \mathrm{~kg}$ de $\mathrm{P}_{2} \mathrm{O}_{5}$ ha $^{-1}$ (fosfato monoamónico); posteriormente, antes del primer riego se aplicaron $90 \mathrm{~kg} \mathrm{~N} \mathrm{ha}^{-1}$, y en el segundo se aplicaron $75 \mathrm{~kg}$ $\mathrm{N} \mathrm{ha}^{-1}$, utilizando en ambos casos sulfato de amonio granulado.

La siembra se realizó manualmente en suelo seco el 10 de octubre de 2012 y el 18 de octubre de 2013. El día de la siembra se aplicó un riego con una lámina de $150 \mathrm{~mm}$. Para facilitar la emergencia de plántulas, se aplicó un riego ligero (60 mm de lámina) a los 16 días después de la siembra (dds) en el primer ciclo y 15 dds en el segundo. El área experimental fue irrigada con el método de riego superficial mediante un sistema de tubos de plástico con compuertas. Durante el ciclo de crecimiento se aplicaron tres riegos, a los 33, 49 y 67 dds en 2012-2013, y a los 35, 50 y 78 dds en 2013-2014; en los dos primeros se utilizó una lámina de $120 \mathrm{~mm}$, mientras que en el tercero se utilizó una lámina ligera $(60 \mathrm{~mm})$. El control de maleza se efectuó en forma manual con azadón. 
En el ciclo 2012-2013 se evaluaron los siguientes cultivares: variedad IMC 205 (Inter. Mountain Cargill), el híbrido Hyola 401 (Interstate Seed Co.), variedad Monty (Dovuron Seeds, Co.) y las variedades Canorte, Ortegón, Aztecan, Canomex y Centenario del INIFAP. En el ciclo 2013-2014 se evaluaron los mismos cultivares primaverales del primer ciclo, más tres variedades tipo invernal: Claremore (Johnston Seed Co.), Sumner (Kansas State Univ.) y Riley (Kansas State Univ.).

Las parcelas experimentales fueron de 10 surcos de 5.0 $\mathrm{m}$ de longitud con un distanciamiento de $0.2 \mathrm{~m}$. Las mediciones se hicieron en seis surcos centrales en un tramo con longitud de $3.0 \mathrm{~m}\left(3.6 \mathrm{~m}^{2}\right)$. Se utilizó un diseño experimental de bloques completos al azar con cuatro repeticiones. En la siembra se utilizaron $12 \mathrm{~kg} \mathrm{ha}^{-1}$ de semilla certificada, con 303,000 a 355,000 semillas kg-1 y un porcentaje de germinación entre 80 y 90 \%. Posteriormente se realizó un aclareo de plantas para establecer una densidad de población de 120 plantas $\mathrm{m}^{-2}$. La cosecha se llevó a cabo entre los 75 y 91 dds en el primer ciclo, y entre los 82 y 106 dds en el segundo, cuando los cultivares de primavera alcanzaron la floración completa (etapa 4.4) (Harper y Berkenkamp, 1975). Los cultivares de invierno que no desarrollaron inflorescencia se cosecharon al mismo tiempo que el cultivar primaveral más tardío (Monty).

En la cosecha se determinaron los rendimientos de forraje fresco y de MS. El contenido de MS se determinó en una muestra de $0.4 \mathrm{~m}^{2}$ tomada al azar de la muestra usada para las mediciones. Para ello, se muestrearon $2 \mathrm{~m}$ de longitud de uno de los surcos centrales de cada parcela. Las plantas muestreadas fueron secadas a $60^{\circ} \mathrm{C}$ en una estufa de aire forzado hasta alcanzar peso constante. El rendimiento de MS se determinó multiplicando el rendimiento de forraje fresco por el contenido de MS de cada parcela.

La distribución de MS en los órganos de la parte aérea se determinó con una muestra al azar de $0.2 \mathrm{~m}^{2}$ tomada de la parcela usada para mediciones; para este propósito se tomó una muestra de $1.0 \mathrm{~m}$ de longitud de uno de los surcos centrales. Después de separar tallos, hojas (lámina y pecíolos) y órganos reproductivos (flores y silicuas), todos ellos se secaron a $60{ }^{\circ} \mathrm{C}$ hasta alcanzar peso constante para determinar el porcentaje de MS asignada a cada órgano de la parte aérea del cultivo.

Las plantas muestreadas para estimar el contenido de MS fueron también usadas para determinar la composición química del forraje en términos de proteína cruda (PC), fibra detergente ácido (FDA), fibra detergente neutro (FDN) y energía neta para lactancia $\left(E N_{1}\right)$. Las plantas fueron molidas en un molino Wiley ${ }^{\circledR}$ (Thomas Scientific, Swedesboro,
NJ, USA) con malla de $1 \mathrm{~mm}$. Las muestras fueron analizadas de acuerdo con el procedimiento descrito por Van Soest et al. (1991) para FDN y FDA, y con el método Kjeldahl para N (Bremner, 1996). El contenido de EN $N_{L}$ se estimó de acuerdo con las metodologías del Consejo Nacional de Investigación (NRC, 2001). Los rendimientos de PC y EN por hectárea se obtuvieron al multiplicar los contenidos de PC y EN rimental.

Los análisis estadísticos para todas las variables se presentan por ciclo. Se realizaron análisis de varianza ( $P$ $\leq 0.05)$ para las siguientes variables: rendimientos de MS, PC y EN ${ }_{L}$, y concentraciones de PC, FDA, FDN y EN $\mathrm{L}_{L}$. Para comparar las medias se utilizó la prueba de la diferencia mínima significativa protegida de Fisher $(P \leq 0.05)$. Se hicieron análisis de regresión lineal simple $(P \leq 0.05)$ para determinar la relación entre la MS asignada a la hoja con los contenidos de PC, FDN y EN $\mathrm{E}_{L}$. El análisis de la información se efectuó con el programa estadístico SAS (SAS Institute, 2011).

\section{RESULTADOS Y DISCUSIÓN}

\section{Características climáticas y agronómicas}

Las condiciones climáticas prevalecientes durante los dos ciclos en que se evaluaron los cultivares de canola fueron diferentes, ya que durante el segundo ciclo de crecimiento se registró una menor temperatura, mayor humedad relativa, menor evaporación y mayor cantidad de lluvia. Las principales diferencias entre ciclos ocurrieron en los meses de noviembre y diciembre (Cuadro 1), cuando los cultivares primaverales estuvieron en las fases de "roseta" (etapa 2.1-2.2) a "floración" (etapa 4.1-4.4) (Harper y Berkenkamp, 1975). Los cultivares tipo invernal no desarrollaron inflorescencia.

\section{Composición química del forraje}

En los dos ciclos de evaluación se registró diferencia significativa $(P \leq 0.05)$ entre cultivares en los parámetros de la composición química del forraje. Los cultivares invernales Claremore, Sumner y Riley se caracterizaron por su mayor contenido de proteína cruda (PC) y energía neta para lactancia $\left(E N_{L}\right)$, y menor concentración de fibra detergente ácido (FDA) y fibra detergente neutro (FDN) al compararse con los cultivares primaverales $(P \leq 0.05)$. En los cultivares primaverales, la concentración de nutrientes fue diferente no sólo entre los nuevos cultivares sino también con respecto a los cultivares testigo. Las principales diferencias se observaron en el contenido de PC (Cuadro 2).

Los nuevos cultivares registraron mayores $(P \leq 0.05)$ o 
Cuadro 1. Elementos del clima durante el desarrollo de cultivares de canola establecidos en dos ciclos de crecimiento en Matamoros, Coahuila, México.

\begin{tabular}{|c|c|c|c|c|c|c|c|}
\hline \multirow{2}{*}{ Periodo } & \multirow{2}{*}{ Ciclo } & \multicolumn{3}{|c|}{ Temperatura media $\left({ }^{\circ} \mathrm{C}\right)$} & \multirow{2}{*}{$\mathrm{HR}^{+}(\%)$} & \multirow{2}{*}{$\mathrm{Epan}^{++}(\mathrm{mm})$} & \multirow{2}{*}{ Lluvia (mm) } \\
\hline & & máximas & mínimas & media & & & \\
\hline \multirow[t]{2}{*}{ Oct. 1-31 } & 2012-2013 & 30.84 & 14.24 & 22.31 & 48.60 & 159.50 & 17.0 \\
\hline & 2013-2014 & 30.01 & 15.35 & 22.50 & 52.42 & 155.50 & 67.8 \\
\hline \multirow[t]{2}{*}{ Nov. 1-30 } & 2012-2013 & 26.11 & 10.40 & 18.12 & 50.52 & 139.42 & 6.6 \\
\hline & 2013-2014 & 22.89 & 9.96 & 16.11 & 63.85 & 127.66 & 21.8 \\
\hline \multirow[t]{2}{*}{ Dic. 1-31 } & 2012-2013 & 24.27 & 7.19 & 15.49 & 36.51 & 146.12 & 0 \\
\hline & 2013-2014 & 22.09 & 6.71 & 14.29 & 51.24 & 141.35 & 7.4 \\
\hline \multirow[t]{2}{*}{ Ene 1-31 } & 2012-2013 & 21.44 & 4.71 & 13.12 & 39.76 & 145.23 & 0 \\
\hline & 2013-2014 & 21.30 & 4.24 & 12.27 & 53.66 & 158.26 & 29.4 \\
\hline
\end{tabular}

${ }^{\dagger}$ HR: humedad relativa; ${ }^{+\dagger}$ Epan: evaporación del tanque evaporímetro.

iguales $(P>0.05)$ contenidos de $P C$ que los observados en los testigos; en los que sobresalieron, en el primer ciclo, Ortegón, Aztecan, Canomex y Monty al compararse con el testigo IMC 205. En el segundo ciclo, los cultivares Canorte, Ortegón y Aztecan presentaron una mayor concentración de PC que IMC 205. En relación a Hyola 401, sólo Monty obtuvo mayores contenidos de PC en el primer ciclo ( $\mathrm{P} \leq$ $0.05)$, los otros nuevos cultivares tuvieron concentraciones similares $(P>0.05)$ (Cuadro 2).

En contenido de fibras, todos los nuevos cultivares primaverales presentaron valores de FDA y FDN similares al testigo IMC 205 ( $P$ > 0.05). En comparación con híbrido Hyola 401, los nuevos cultivares tuvieron mayores ( $P \leq$ $0.05)$ o iguales valores $(P>0.05)$ de contenido fibroso. El cultivar Canomex mostró valores similares de FDA en los dos ciclos, mientras que Aztecan y Monty tuvieron valores iguales sólo en el primero. En contenido de FDN, los cultivares Ortegón, Aztecan y Canomex presentaron valores estadísticamente iguales $(P>0.05)$ al híbrido Hyola 401 en los dos ciclos; mientras que los cultivares Centenario y Monty obtuvieron valores iguales en el segundo y primer ciclo, respectivamente (Cuadro 2).

En cuanto al contenido de $\mathrm{EN}_{\mathrm{L}^{\prime}}$ en el primer ciclo los nuevos cultivares primaverales Canomex y Monty fueron sobresalientes, con valores estadísticamente iguales ( $P$ > 0.05) al registrado en el híbrido Hyola 401; para el segundo ciclo destacaron los cultivares invernales $(P \leq 0.05)$, seguidos de los cultivares primaverales Hyola 401, Aztecan y Canomex, estos tres últimos fueron estadísticamente iguales $(P>0.05)$ (Cuadro 2).

En el primer ciclo de evaluación, las diferencias entre cultivares primaverales en los parámetros de la composición química, no estuvieron relacionadas con la distribución de MS en los órganos de la parte aérea (hoja, tallo y silicuas), como lo indica la falta de significancia estadística $(P$ > 0.05) en los parámetros de la regresión entre el porcentaje de MS asignada a la hoja (\% MS hoja) y los valores de PC,

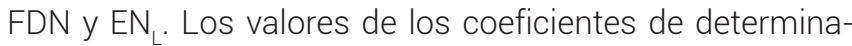
ción $\left(R^{2}\right)$ y los de la probabilidad $(P)$ de que la pendiente de la recta de regresión es significativamente diferente de cero $(P \leq 0.05)$ fueron los siguientes: \% MS hoja- $P C, R^{2}=$ $0.043, P=0.621 ; \%$ MS hoja-FDN, $R^{2}=0.044 ; P=0.619 ; \%$ MS hoja- $E N_{L^{\prime}} R^{2}=0.077 ; P=0.507$

En el segundo ciclo cuando se evaluaron los cultivares primaverales junto con los cultivares invernales, se encontró una relación significativa $(P \leq 0.05)$ entre el porcentaje de MS distribuida en la hoja y los parámetros de la composición química del forraje. En esta relación se observa que los cultivares invernales presentaron las mayores concentraciones de PC y EN $\mathrm{L}^{\prime}$ y menor contenido fibroso (Figura 1), debido a que no desarrollaron inflorescencia, además de que asignaron una mayor proporción de MS a la hoja (52.3 a $58.3 \%$ ) y menor hacia el tallo (41.6 a $47.6 \%$ ) en comparación con los cultivares primaverales, los cuales registraron porcentajes de MS en la hoja de 31.7 a $43.4 \%$, de 42.7 a $61.7 \%$ en el tallo y de 7.2 a $13.9 \%$ en órganos reproductivos. Esta respuesta puede explicarse por el mayor contenido de PC en hojas (200.0 a $250 \mathrm{~g} \mathrm{~kg}^{-1}$ ) con respecto al tallo (100.0 $\left.\mathrm{g} \mathrm{kg}^{-1}\right)$ de acuerdo con lo observado por Rao y Horn (1995); y la menor concentración de FDN en hoja $\left(208.0 \mathrm{~g} \mathrm{~kg}^{-1}\right)$ con respecto a la del tallo $\left(480.0 \mathrm{~g} \mathrm{~kg}^{-1}\right)$ como lo reportaron Chapman et al. (2009).

Las concentraciones de nutrientes observados en los cultivares primaverales se encuentran dentro de los rangos observados en otros trabajos, con algunas variaciones de acuerdo con la fase del desarrollo en que se realizó la cosecha y el manejo agronómico del cultivo. En la Comarca Lagunera de México, el forraje de canola cosechada en floración plena (etapa 4.2) o al final de floración (etapa 4.4) 
Cuadro 2. Concentración de proteína cruda (PC), fibra detergente ácido (FDA), fibra detergente neutro (FDN) y energía neta para lactancia $\left(E_{\mathrm{L}}\right)$ en el forraje de cultivares de canola evaluados en dos ciclos en Matamoros, Coahuila, México.

\begin{tabular}{|c|c|c|c|c|c|c|c|c|}
\hline \multirow{2}{*}{ Cultivares } & \multicolumn{2}{|c|}{$P C\left(g_{k g}^{-1}\right)$} & \multicolumn{2}{|c|}{ FDA $\left(\mathrm{g} \mathrm{kg}^{-1}\right)$} & \multicolumn{2}{|c|}{ FDN $\left(\mathrm{g} \mathrm{kg}^{-1}\right)$} & \multicolumn{2}{|c|}{$\mathrm{EN}_{\mathrm{L}}\left(\mathrm{MJ} \mathrm{kg}^{-1} \mathrm{MS}^{\ddagger}\right)$} \\
\hline & 2012-2013 & 2013-2014 & 2012-2013 & 2013-2014 & 2012-2013 & 2013-2014 & 2012-2013 & 2013-2014 \\
\hline IMC 205 & $167.9 c^{\dagger}$ & $159.0 \mathrm{f}$ & 338.8 ab & $368.8 \mathrm{ab}$ & $420.4 \mathrm{abc}$ & 495.6 a & 5.90 bc & $5.41 \mathrm{~cd}$ \\
\hline Hyola 401 & 177.5 bc & 173.6 cdef & $310.3 \mathrm{c}$ & $329.9 \mathrm{c}$ & 395.8 bc & $440.3 b$ & $6.23 \mathrm{a}$ & $5.86 b$ \\
\hline Canorte & 181.6 bc & $180.6 \mathrm{~cd}$ & 339.6 ab & 385.1 a & $433.2 \mathrm{abc}$ & 506.1 a & 5.90 bc & $5.22 \mathrm{~d}$ \\
\hline Ortegón & $188.7 \mathrm{ab}$ & $185.9 \mathrm{c}$ & 358.9 a & $362.2 \mathrm{ab}$ & $438.5 \mathrm{ab}$ & $464.9 \mathrm{ab}$ & $5.69 \mathrm{c}$ & $5.49 \mathrm{~cd}$ \\
\hline Aztecan & 186.5 b & 177.5 cde & $346.8 \mathrm{ab}$ & 352.6 bc & $408.1 \mathrm{abc}$ & $474.8 \mathrm{ab}$ & $5.82 \mathrm{bc}$ & $5.60 \mathrm{bc}$ \\
\hline Canomex & $191.0 \mathrm{ab}$ & 173.6 cdef & 328.8 bc & 351.8 bc & $395.0 \mathrm{c}$ & $464.3 \mathrm{ab}$ & $6.02 a b$ & $5.61 \mathrm{bc}$ \\
\hline Centenario & 180.0 bc & 167.7 def & $353.8 \mathrm{ab}$ & $362.2 \mathrm{ab}$ & $441.0 \mathrm{a}$ & $465.8 \mathrm{ab}$ & 5.73 bc & $5.49 \mathrm{~cd}$ \\
\hline Monty & $201.0 \mathrm{a}$ & $164.2 \mathrm{ef}$ & 329.8 bc & $386.4 \mathrm{a}$ & $417.6 \mathrm{abc}$ & $510.0 \mathrm{a}$ & $6.02 a b$ & $5.21 \mathrm{~d}$ \\
\hline Claremore & - & $230.1 \mathrm{a}$ & - & $243.0 \mathrm{~d}$ & - & $323.9 \mathrm{c}$ & - & $6.85 a$ \\
\hline Sumner & - & $220.0 \mathrm{ab}$ & - & $254.7 \mathrm{~d}$ & - & $320.0 \mathrm{c}$ & - & $6.72 \mathrm{a}$ \\
\hline Riley & - & $214.5 b$ & - & $246.6 d$ & - & $322.4 \mathrm{c}$ & - & $6.81 \mathrm{a}$ \\
\hline
\end{tabular}

${ }^{\top}$ Medias con la misma letra en las columnas no son estadísticamente diferentes (DMS $\left.\leq 0.05\right) ;{ }^{\ddagger}$ MS: materia seca.

presentó concentraciones de PC de 159.0 a $280.0 \mathrm{~g} \mathrm{~kg}^{-1}$, 340 a $433 \mathrm{~g} \mathrm{~kg}^{-1}$ en FDA, 389 a $466.0 \mathrm{~g} \mathrm{~kg}^{-1}$ de FDN y 3.7 a $6.1 \mathrm{MJ} \mathrm{kg}^{-1} \mathrm{MS}$ en EN (Cruz et al., 2012; Reta et al., 2008; Reta et al., 2010; Reta et al., 2015; Reta et al., 2016; RetaSánchez et al., 2016a, 2016b).

En los cultivares tipo invernal, los contenidos de PC (220.0 a $\left.230.1 \mathrm{~g} \mathrm{~kg}^{-1}\right)$, FDA (243.0 a $254.7 \mathrm{~g} \mathrm{~kg}^{-1}$ ) y FDN (320.0 a $323.9 \mathrm{~g} \mathrm{~kg}^{-1}$ ) observados en el estudio fueron ligeramente mayores a los reportados por Neely et al., (2009) en E.E.U.U., los cuales fueron $211.0 \mathrm{~g} \mathrm{~kg}^{-1}$ en PC, $199.0 \mathrm{~g}$ $\mathrm{kg}^{-1}$ en FDA y $207.0 \mathrm{~g} \mathrm{~kg}^{-1}$ en FDN.

\section{Ciclo de crecimiento y rendimiento de materia seca y nutrientes}

La presencia de bajas temperaturas durante el segundo ciclo del estudio (Cuadro 1) alargó el periodo de crecimiento de los cultivares de primavera. Los nuevos cultivares de primavera, con excepción de Monty, fueron entre 6 y 16 d más precoces que los testigos IMC 205 y Hyola 401 en los dos ciclos (Cuadro 3).

En los dos ciclos de evaluación se encontraron diferencias significativas entre cultivares $(P \leq 0.05)$ en potencial de rendimiento de MS y nutrientes. Los de mayor rendimiento fueron los testigos IMC 205 y Hyola 401, y tres de los nuevos cultivares evaluados, Ortegón, Monty y Claremore. Entre estos cultivares sobresalientes, Ortegón presentó la ventaja adicional de una mayor precocidad, entre 6 y $16 \mathrm{~d}$ con respecto a IMC 205 y Hyola 401 (Cuadro 3). Esta precocidad es importante para la integración de los cultivares de canola en los sistemas de producción intensivos de fo- rraje en la región, ya que permite obtener dos cosechas en el ciclo otoño-invierno, o bien la liberación más temprana del terreno para las siembras de primavera y el ahorro de un riego con respecto a la avena (Reta et al., 2016).

El cultivar de mayor estabilidad en rendimiento fue la variedad IMC 205, seguida del híbrido Hyola 401, que sólo fue inferior en rendimiento de MS ( $P \leq 0.05)$ en el segundo ciclo. El cultivar Ortegón fue igual $(P>0.05)$ en todos los componentes a Hyola 401, e inferior $(P \leq 0.05)$ en rendimiento de MS y EN $N_{L}$ con respecto a IMC 205 en el segundo ciclo. Los cultivares precoces Canorte, Aztecan, Canomex y Centenario mostraron rendimientos de MS entre el 72.1 y $80.8 \%$ en el primer ciclo, y entre el 53.4 y $75.6 \%$ en el segundo en comparación con los rendimientos de MS obtenidos en el testigo IMC 205 (Cuadro 3).

El rendimiento de MS y nutrientes en los cultivares primaverales sobresalientes fue de 7216 a $8480 \mathrm{~kg} \mathrm{ha}^{-1}$ de MS, de 1270 a $1456 \mathrm{~kg} \mathrm{ha}^{-1}$ de PC y de 39,663 a 47,928 MJ $\mathrm{ha}^{-1}$ en EN $\mathrm{L}_{\text {. }}$ En los cultivares de invierno, los rendimientos de MS fueron entre 26.7 y $69.4 \%$ inferiores (5006 a 6215 $\mathrm{kg} \mathrm{ha}^{-1}$ ) a los del testigo IMC 205 (Cuadro 3); sin embargo, debido al mayor contenido de PC y EN $\mathrm{E}_{\mathrm{L}}$ en su forraje (Cuadro 2), sus niveles de rendimiento en estos nutrientes fueron similares a los obtenidos por los mejores cultivares primaverales. De estos cultivares, Claremore fue sobresa-

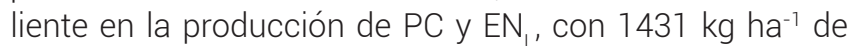
$P C$ y de $42,625 \mathrm{MJ} \mathrm{ha}^{-1}$ en $E N_{\llcorner}$. Estos rendimientos fueron estadísticamente iguales $(P>0.05)$ a los testigos IMC 205 y Hyola 401. El cultivar Riley obtuvo rendimientos de PC y EN $\mathrm{L}_{\llcorner}$similares $(P>0.05)$ a Hyola 401 (Cuadro 3). 

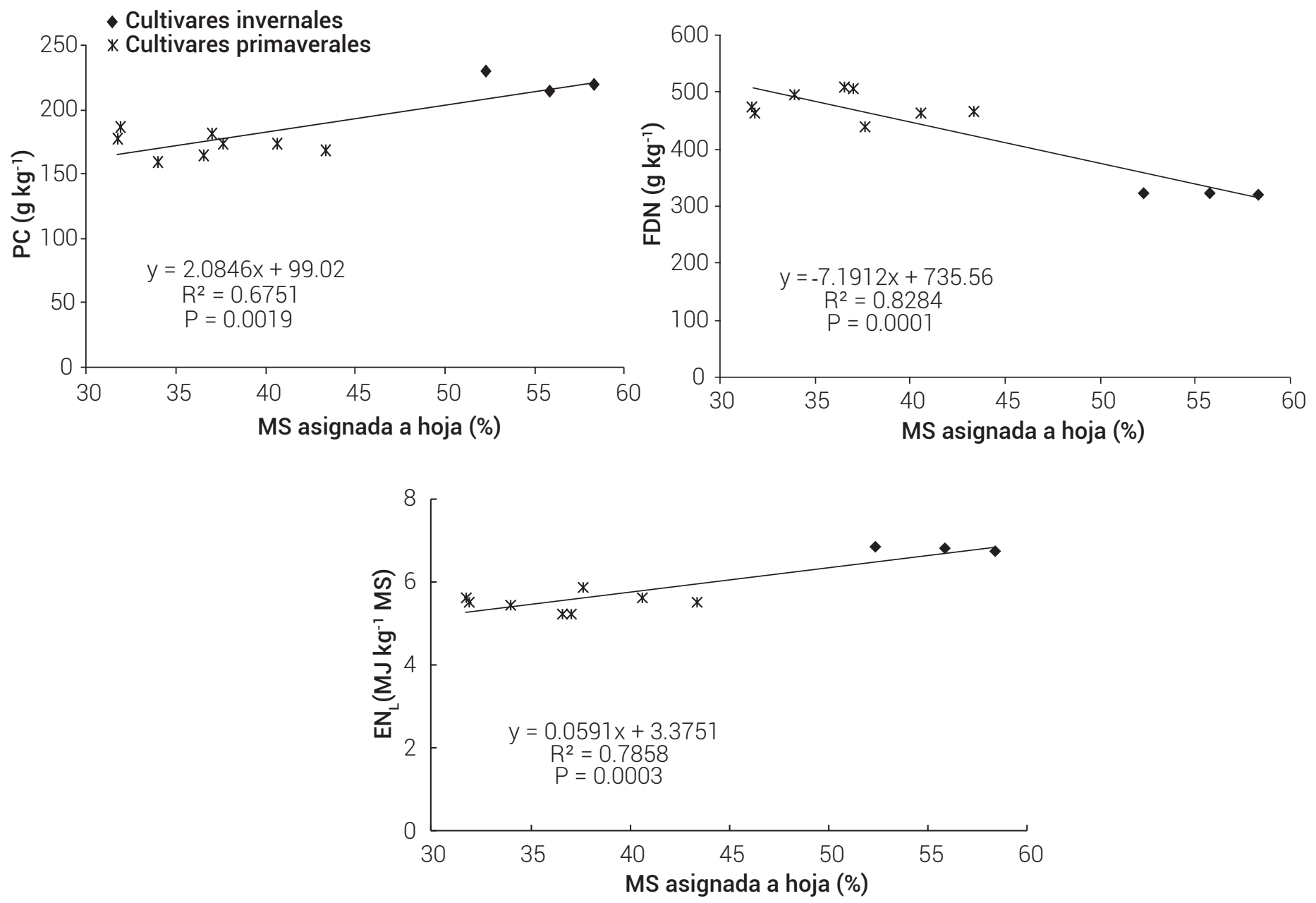

Figura 1. Relación entre el porcentaje de materia seca (MS) asignada a hoja y los contenidos de proteína cruda (PC), fibra detergente neutro (FDN) y energía neta para lactancia $\left(E N_{\mathrm{L}}\right)$ en el forraje de cultivares de canola tipo primaveral e invernal evaluados durante el ciclo 2013-2014 en Matamoros, Coahuila, México. P : probabilidad de que la pendiente de la recta de regresión sea significativamente diferente de cero.

Los rendimientos de MS y nutrientes obtenidos fueron similares o menores a los encontrados en varios trabajos realizados en la Comarca Lagunera. En estos trabajos previos, los rendimientos de MS con cultivares primaverales fluctuaron de 5311 a 10,300 kg ha-1, de 978 a $2486 \mathrm{~kg} \mathrm{ha}^{-1}$ en PC y de 24,835 a 47,928 $\mathrm{MJ} \mathrm{ha}^{-1}$ en $\mathrm{EN}_{1}$, de acuerdo con el clima y manejo agronómico del cultivo (Cruz et al., 2012; Reta et al., 2008; Reta et al., 2010; Reta et al., 2015; Reta et al., 2016; Reta-Sánchez et al., 2016a, 2016b). En cultivares tipo invernal establecidos en Australia y Estados Unidos (E.E.U.U) se han obtenido rendimientos de MS similares a los obtenidos en el presente estudio, los que alcanzaron entre 3000 y $6800 \mathrm{~kg} \mathrm{ha}^{-1}$ (Neely et al., 2009; Sprague et al., 2015).

\section{CONCLUSIONES}

El rendimiento de MS y el contenido de nutrientes en el forraje de los nuevos cultivares primaverales Ortegón y Monty, además de una mayor precocidad en el cultivar Ortegón, generan la posibilidad de integrarlos en los sistemas de producción de forraje en la región. Con respecto a los cultivares de invierno, Claremore por sus rendimientos de PC y EN, comparables con los mejores cultivares de primavera también puede ser integrado en los sistemas de producción de forraje.

\section{AGRADECIMIENTOS}

Al Instituto Nacional de Investigaciones Forestales, Agrícolas y Pecuarias por el financiamiento de este estudio.

\section{BIBLIOGRAFÍA}

Bremner J. M. (1996) Nitrogen-total. In: Methods of Soil Analysis. Part 3. Chemical Methods. D. L. Sparks (ed.). SSSA Book Series 5. Soil Science Society of America. Madison, WI. USA. pp:1085-1121. 
Cuadro 3. Ciclo de crecimiento y rendimientos de materia seca (MS), proteína cruda (PC) y energía neta para lactancia $\left(\mathrm{EN}_{\mathrm{L}}\right)$ de cultivares de canola evaluados en dos ciclos en Matamoros, Coahuila, México.

\begin{tabular}{|c|c|c|c|c|c|c|c|c|}
\hline \multirow[t]{2}{*}{ Cultivares } & \multicolumn{2}{|c|}{ Ciclo (días) } & \multicolumn{2}{|c|}{$\mathrm{MS}\left(\mathrm{kg} \mathrm{ha}^{-1}\right)$} & \multicolumn{2}{|c|}{$\mathrm{PC}\left(\mathrm{kg} \mathrm{ha}^{-1}\right)$} & \multicolumn{2}{|c|}{$\mathrm{EN}_{\mathrm{L}}\left(\mathrm{MJ} \mathrm{ha}^{-1}\right)$} \\
\hline & 2012-2013 & 2013-2014 & 2012-2013 & 2013-2014 & 2012-2013 & 2013-2014 & 2012-2013 & 2013-2014 \\
\hline IMC 205 & 91 & 97 & $8129 a^{\dagger}$ & $8480 \mathrm{a}$ & $1367 \mathrm{ab}$ & $1349 a b$ & $47,928 \mathrm{a}$ & 45,863 a \\
\hline Hyola 401 & 86 & 97 & $7407 \mathrm{ab}$ & $7302 b$ & $1315 a b c$ & $1270 \mathrm{abc}$ & $46,111 \mathrm{a}$ & $42,719 a b$ \\
\hline Canorte & 75 & 88 & 6573 bc & 5485 efg & 1192 bc & 991 ef & $38,790 \mathrm{bcd}$ & 28,587 ef \\
\hline Ortegón & 75 & 91 & $7326 a b$ & 7216 bc & $1388 a b$ & $1343 a b$ & $41,705 a b c$ & 39,663 bc \\
\hline Aztecan & 75 & 85 & 5859 c & $4862 \mathrm{gh}$ & $1091 \mathrm{c}$ & $861 \mathrm{fg}$ & 34,005 d & $27,231 \mathrm{f}$ \\
\hline Canomex & 75 & 85 & 5916 c & $6414 \mathrm{~cd}$ & $1130 \mathrm{c}$ & 1117 cde & $35,628 \mathrm{~cd}$ & $36,005 \mathrm{~cd}$ \\
\hline Centenario & 75 & 82 & $6069 c$ & $4529 \mathrm{~h}$ & $1093 \mathrm{c}$ & $760 \mathrm{~g}$ & $34,845 d$ & $24,835 f$ \\
\hline Monty & 86 & 106 & $7258 a b$ & $6388 \mathrm{~d}$ & $1456 a$ & 1052 def & $43,617 a b$ & 33,483 de \\
\hline Claremore & - & 106 & - & 6215 de & - & 1431 a & - & $42,625 a b$ \\
\hline Sumner & - & 106 & - & $5006 \mathrm{fgh}$ & - & 1100 cde & - & 33,615 de \\
\hline Riley & - & 106 & - & 5736 def & - & 1230 bcd & - & 39,048 bcd \\
\hline
\end{tabular}

${ }^{\top}$ Medias con la misma letra en las columnas no son estadísticamente diferentes (DMS $\left.\leq 0.05\right)$.

Brennan R. F. and M. D. A. Bolland (2001) Comparing fertilizer phosphorus requirements of canola, lupin, and wheat. Journal of Plant Nutrition 24:1885-1900.

Chapman G., E. Bork, N. Donkor and R. Hudson (2009) Yields, quality and suitability of four annual forages for deer pasture in North Central Alberta. The Open Agriculture Journal 3:26-31.

Cruz C. J. J., G. Núñez H., R. Faz C., D. G. Reta S. y H. A. Serrato M. (2012) Potencial forrajero y eficiencia de uso del aqua de canola (Brassica napus L.) en comparación con cultivos tradicionales en el ciclo de invierno. Agrofaz 12:125-130.

Fiebelkorn D. and M. Rahman (2016) Development of a protocol for frosttolerance evaluation in rapeseed/canola (Brassica napus L.). The Crop Journal 4:147-152.

García M. K. P., A. González Á. y N. Castillo T. (2013) Tecnología para producir canola de temporal en las Sierras de Tapalpa y el Tigre en Jalisco. Folleto para Productores Núm. 1. SAGARPA-INIFAPCIRPAC-Campo Experimental Centro Altos de Jalisco. Tepatitlán de Morelos, Jalisco, México. 31 p.

González Q. J. y A. S. Ortegón M. (2013) Canorte 2010. Nueva variedad de canola mexicana. Ficha de la tecnología validada en 2012. In: Tecnologías Generadas, Validadas o Transferidas en los estados de Tamaulipas, San Luis Potosí, Coahuila y Nuevo León en el Año de 2012. J. Elizondo B., I. H. Almeyda L., J. L. Barrón C., L. M. Torres E. y G. J. García D. (eds.). Folleto Técnico No. MX-0310301-52-03-13-09-59. SAGARPA-INIFAP-CIRNE. Río Bravo, Tamaulipas. pp:73-74

Harper F. R. and B. Berkenkamp (1975) Revised growth-stage key for Brassica campestris and B. napus. Canadian Journal of Plant Science 55:657-658.

Hernández R., A. González y P. Rivera (2008) El cultivo de la canola (Brassica napus y B. rapa) en el estado de Jalisco, México. I. Características agronómicas. Bioagro 20:185-191.

Hernández R., P. Rivera y A. González (2009) El cultivo de la canola (Brassica napus y B. rapa) en el estado de Jalisco, México. II. Ventajas económicas sobre el cultivo del trigo. Bioagro 21:63-67.

Ortegón M. A. S., A. Díaz F., J. González Q. e I. Garza C. (2006) La temperatura en la etapa reproductiva del cultivo de canola (Brassica napus L.). Agricultura Técnica en México 32:259-265.

Ortegón-Morales A. S., A. Díaz-Franco y J. González-Quintero (2007) Cultivares de canola y su interacción con el ambiente y el método de siembra. Universidad y Ciencia 23:21-28.

Neely C., J. Brown, C. Hunt and J. Davis (2009) Increasing the value of winter canola crops by developing ensiling systems (canolage) to produce cattle feed. In: Idaho Alfalfa and Forage Conference Proceedings. 3-4 February 2009, Burley, Idaho, USA. University of Idaho Extension. Burley, Idaho, USA. pp:27-31.
NRC, National Research Council (2001) Nutrient Requirements of Dairy Cattle. National Academy Press. Washington, D.C. USA. 408 p.

Paridaen A. and J. A. Kirkegaard (2015) Forage canola (Brassica napus): spring-sown winter canola for biennial dual-purpose use in the high-rainfall zone of southern Australia. Crop and Pasture Science 66:275-286.

Purty R. S., G. Kumar, S. L. Singla-Pareek and A. Pareek (2008) Towards salinity tolerance in Brassica: an overview. Physiology and Molecular Biology of Plants 14:39-49.

Rao S. C. and F. P. Horn (1995) Cereals and brassicas for forage. In: Forages. Volume I. R. F. Barnes, D. A. Miller and C. J. Nelson (eds.) lowa State University Press. Ames, lowa. pp:451-462.

Reta S. D. G., J. S. Serrato C., A. G. Gaytán M., H. M. Quiroga, F. Frausto L., S. Ordaz V. y J. Godoy H. (2016) Validación de patrones de cultivo forrajeros alternativos con menor estrés por altas temperaturas en la Comarca Lagunera. Agrofaz 16:65-75.

Reta-Sánchez D. G., J. S. Serrato-Corona, H. M. Quiroga-Garza, A. GaytánMascorro and U. Figueroa-Viramontes (2016a) Forage yield and chemical composition of canola (Brassica napus L.) as affected by sowing methods. Grass and Forage Science 71:281-290.

Reta-Sánchez D. G., J. S. Serrato-Corona, H. M. Quiroga-Garza, U. FigueroaViramontes, A. Gaytán-Mascorro y J. A. Cueto-Wong (2016b) Respuesta de canola para forraje a la densidad de población. Revista Fitotecnia Mexicana 39:253-258

Reta S. D. G., J. S. Serrato C., R. Figueroa V., J. A Cueto W., S. Berúmen P. y J. Santamaría C. (2008) Cultivos Alternativos con Potencial de Uso Forrajero en la Comarca Lagunera. Libro Técnico Núm. 3. INIFAP-CIRNOC-CELALA. Torreón, Coahuila, México. 268 p.

Reta S. D. G., U. Figueroa V., J. S. Serrato C., H. M. Quiroga G., A. Gaytán M. y J. A. Cueto W. (2015) Potencial forrajero y productividad del aqua en patrones de cultivos alternativos. Revista Mexicana de Ciencias Pecuarias 6:153-170.

Reta S. D. G., U. Figueroa V., R. Faz C., G. Núñez H., A. Gaytán M., J. S. Serrato C. y J. A. Payán G. (2010) Sistemas de producción de forraje para incrementar la productividad del agua. Revista Fitotecnia Mexicana 33:83-87.

SAS Institute (2011) The SAS System for Windows, Release 9.3. Statistical Analysis Systems Inst. Cary, NC.

Sprague S. J., J. A. Kirkegaard, J. M. Graham, L. W. Bell, M. Seymour and M. Ryan (2015) Forage and grain yield of diverse canola (Brassica napus) maturity types in the high-rainfall zone of Australia. Crop and Pasture Science 66:260-274.

Van Soest P. J., J. B. Robertson and B. A. Lewis (1991) Methods for dietary fiber, neutral detergent fiber and non-starch polysaccharides in relation to animal nutrition. Journal of Dairy Science 74:3583-3597. 
